\title{
Experimental observation of subwavelength localization using metamaterial-based cavities
}

\author{
Humeyra Caglayan, ${ }^{1, *}$ Irfan Bulu, ${ }^{2}$ Marko Loncar, ${ }^{2}$ and Ekmel Ozbay ${ }^{1}$ \\ ${ }^{1}$ Nanotechnology Research Center-NANOTAM, Department of Physics, Department of Electrical and Electronics \\ Engineering, Bilkent University, Bilkent, 06800 Ankara, Turkey \\ ${ }^{2}$ School of Engineering and Applied Sciences, Harvard University, 33 Oxford Street, Cambridge, \\ Massachusetts 02138, USA \\ *Corresponding author: caglayan@fen.bilkent.edu.tr
}

Received August 5, 2008; revised November 11, 2008; accepted November 23, 2008; posted December 3, 2008 (Doc. ID 99793); published December 24, 2008

\begin{abstract}
We report subwavelength localization of electromagnetic fields within cavities based on metamaterials. Cavity resonances are observed in the transmission spectrum of a split-ring resonator and composite metamaterials cavity structures. These cavity resonances are shown to exhibit high-quality factors. Since the unit cells of metamaterials are much smaller than the operation wavelength, subwavelength localization is possible within these metamaterial cavity structures. In the present Letter, we show that the electromagnetic field is localized into a region of $\lambda / 8$, where $\lambda$ is the cavity resonance wavelength. (C) 2008 Optical Society of America

OCIS codes: $350.4010,160.3918$.
\end{abstract}

The response of any material to electromagnetic (EM) waves is determined by two parameters: dielectric permittivity $(\epsilon)$ and magnetic permeability $(\mu)$. Generally, $\mu$ of naturally occurring materials is positive. Four decades ago, Veselago [1] proposed that artificially constructed materials can enable the negative values of $\epsilon$ and $\mu$, since there are no restrictions with the laws of electromagnetism. In this pioneering study, Veselago predicted that via artificially constructed materials, along with simultaneously negative $\epsilon$ and $\mu$, difficulty in obtaining negative refraction, reverse Doppler shift, and backward Cherenkov radiation can all be overcome. However, obtaining a simultaneously negative $\epsilon$ and $\mu$ and, therefore, a negative index of refraction is a significant challenge. Metallic thin wires arranged periodically are good candidates for a negative $\epsilon$ medium, because this structure exhibits a plasma frequency below which the material is opaque [2]. On the other hand, constructing a negative $\mu$ medium is difficult because of the absence of a magnetic charge. Pendry et al. [3] proposed in 1999 that split-ring resonator (SRR) structures exhibit negative $\mu$. Thereafter, composite metamaterials (CMMs) with simultaneously negative $\epsilon$ and $\mu$ were constructed with a combination of wire and SRR structures [4]. Since then many experimental and theoretical works that have been reported were conducted for novel applications, such as negative refraction [5], subwavelength focusing [6], cloaking [7], and reverse Doppler shift [8].

In addition to the negative index of refraction, metamaterials have another important property: The unit cells of metamaterials are much smaller than the operating wavelength. This property can also lead to different novel applications, such as the localization of the field into a subwavelength region. Recently, the localization of the field has attained great interest from the scientific community, since it is the key issue of several applications. One method for obtaining a localized field is to make a deformation in a unit cell of the periodic structure. This phenomenon has been investigated experimentally and theoretically in photonic crystal structures [9-11]. Photonic crystal defects have many important applications such as thresholdless semiconductor lasers [12] and single-mode light-emitting diodes [13]. However, defect structures in metamaterials are investigated only for periodically arranged single negative materials [14]. In the present Letter, we investigate cavity formation in SRR and CMM structures. We first present the transmission results for SRR and CMM structures. Subsequently, we introduce the cavity structure and present the transmission of the SRR cavity and CMM cavity structures. Our results show that the modification of a unit cell of the metamaterial can exhibit a cavity resonance. Finally, we show that the cavity formation in the metamaterials leads to the localization of the field within a subwavelength region.

The SRR structure that we used for the present study was a one-dimensional periodic arrangement of square rings. The CMM structure was obtained by the combination of the SRR structure and wire stripes. The structures were printed on a Teflon substrate with $\epsilon=2.17$, in which the thickness of the substrate was $1 \mathrm{~mm}$. The wire stripes were on the back of the substrate, and the square SRRs were on the front faces. The thickness of the copper was $0.05 \mathrm{~mm}$. The width of the wire stripes was $1.6 \mathrm{~mm}$. The lattice constant along the $x$ direction (propagation direction) was $4.95 \mathrm{~mm}$. There were five layers along the propagation direction, in which the height of the structure was 40 layers. Thirty layers of structures were stacked with a $2 \mathrm{~mm}$ period along the $z$ direction. The $E$ field was in the $y$ direction. The details regarding the unit cell of the metamaterial structures are shown in Figs. 1(a) and 1(b). The experimental setup consisted of an HP 8510C network analyzer and two standard gain horn antennae in order to measure the transmission amplitude. The calculations throughout 


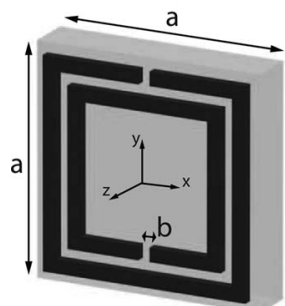

(a)

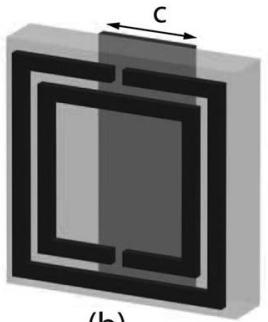

(b)

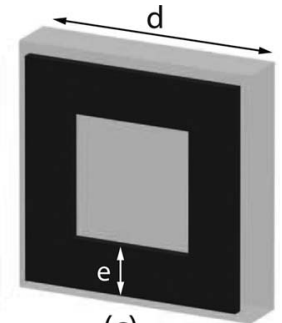

(c)
Fig. 1. (a) Unit cell of the SRR structure: $a=4.95 \mathrm{~mm}, \mathrm{~b}$ $=0.25 \mathrm{~mm}$. (b) Unit cell of the CMM structure: $\mathrm{c}=1.6 \mathrm{~mm}$. (c) Cavity structure: $\mathrm{d}=5.4 \mathrm{~mm}, \mathrm{e}=1 \mathrm{~mm}$. The unit cells of metamaterials are much smaller than the operating wavelength.

the Letter were performed by the commercial software program CST Microwave Studio.

The SRR structure that was used in this work has a bandgap between 5 and $7 \mathrm{GHz}$. However, the closed-ring resonator (CRR) structure, which was obtained by closing the splits in the rings, transmitted EM waves at these frequencies [Fig. 2(a)]. Therefore, this gap is due to the magnetic resonance [15], in which the SRR structure exhibits a negative $\mu$ medium. When the electromagnetic field passes through the ring, an induced current is created, and the generated field is perpendicular to the magnetic field of the light. The magnetic resonance results in a negative $\mu$. EM waves cannot propagate in the negative $\mu$ medium and possess a bandgap in the spectrum. On the other hand, the CMM structure transmits EM waves at the bandgap frequency of the SRR [Fig. 2(b)], since it has negative $\mu$ and ( $\epsilon$ ) at this range [4].

To break the symmetry of these periodic lefthanded metamaterials, we changed the center unit cell of the structures by a closed-ring structure, which was placed on both sides of the board and possessed positive $\epsilon$ and $\mu$ [Fig. 1(c)]. This deformation in the SRR and CMM structures resulted in a cavity structure with a cavity resonance in the transmission spectrum. In Figs. 3(a) and 3(b) the transmission from the SRR cavity and CMM cavity structures are shown, respectively. The calculations agreed well with the experiments. We observed a cavity resonance with the $Q$ factor (quality factor, defined as the center frequency, divided by the FWHM) of 192 at

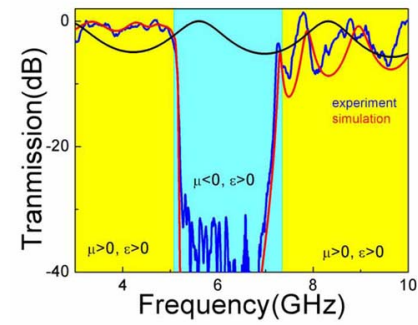

(a)

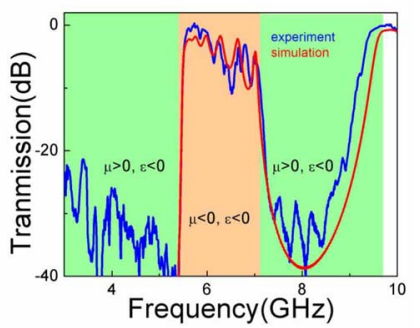

(b)
Fig. 2. (Color online) (a) The SRR structure has a bandgap between 5 and $7 \mathrm{GHz}$. However, the CRR structure transmits EM waves (black curve). Hence, the SRR structure exhibits $\mu<0$ medium at these frequencies. (b) The CMM structure transmits EM waves, because it has $\mu<0$ and $\epsilon<0$ at this range.

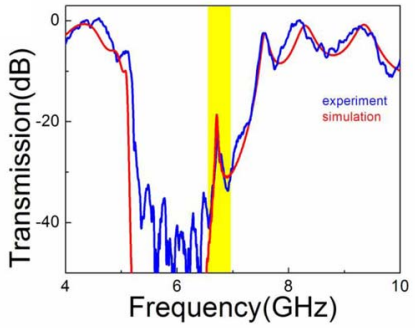

(a)

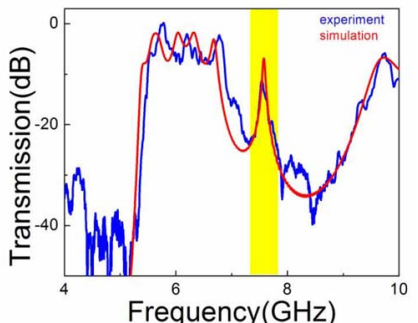

(b)
Fig. 3. (Color online) A cavity structure is introduced by replacing the center unit cell with a positive-index medium. The cavity resonance is observed at $6.7 \mathrm{GHz}$ $(44.7 \mathrm{~mm})$ and $7.5 \mathrm{GHz}(40 \mathrm{~mm})$ by the (a) SRR cavity structure and (b) the CMM cavity structure, respectively.

$6.7 \mathrm{GHz}(44.7 \mathrm{~mm})$ by the SRR cavity structure. The cavity resonance of the CMM structure was obtained at $7.5 \mathrm{GHz}(40 \mathrm{~mm})$ with a $Q$ factor of 108 .

The reflection of the SRR (CMM) structure is very high in the negative $\mu(\epsilon)$ frequency range, and, therefore, the SRR (CMM) structures on both sides of the cavity behave like frequency-specific mirrors $[16,17]$. Any propagating light that is trapped between them will bounce back and forth between these two mirrors. Since the mirrors localize light within a finite region, the modes are quantized into discrete frequencies, just as in Fabry-Perot resonances. The cavity resonance frequency depends on the geometry and size of the defects, as well as the geometry of the SRR and CMM. This mechanism is investigated in [18].

The calculated electric-field distributions for the cavity structures show that the EM waves at the cavity resonance are trapped at the positive index region (Fig. 4). The length of the cavity is only $\lambda / 8$, where $\lambda$ is the cavity resonance wavelength. Hence, the field at the cavity resonance is enhanced at the subwavelength cavity region. Such a structure with enhanced electromagnetic fields can be used for several applications, including nonlinear optics. Although it is possible to localize light into a subwavelength region with a regular Fabry-Perot cavity, this localization is not smaller than $\lambda / 2 \mathrm{n}$, where $n$ is the refractive index of the dielectric gap. However, the phase dispersion properties of metamaterial-based cavity systems can be used to confine light into even smaller subwavelength dimensions.

Although the field is highly localized at the cavity region, the quality factors are not that high. The calculated transmissions for an SRR cavity structure

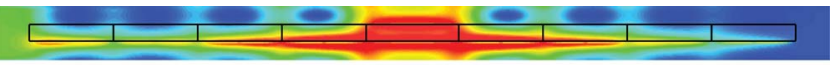

(a)

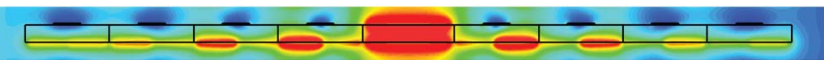

(b)

Fig. 4. (Color online) The calculated electric field is highly localized at the cavity region for (a) SRR cavity and (b) CMM cavity structures. Hence, the field at the cavity resonance is enhanced at the subwavelength $(\lambda / 8)$ cavity region. (Red indicates the maximum, and blue indicates the minimum.) 
without loss, with loss on a board only, and loss on metal only showed that the transmission at the cavity resonance is decreased because of the loss of the board and metal $(\mathrm{Cu})$. The calculated $Q$ factor for the SRR cavity resonance without loss is 3290 , whereas for the CMM cavity it is 1420 . Therefore, one can obtain metamaterial cavities with higher $Q$ factors by using different designs and materials.

In conclusion, we report what we believe to be the first experimental observation of cavity formation in metamaterials. Our results showed that it is possible to obtain a cavity structure by the deformation of a unit cell of metamaterials. We presented the $Q$ factors of the cavity resonances as 192 for an SRR-based cavity and 108 for a CMM-based cavity. Furthermore, it is possible to obtain a $Q$ factor as high as 3290 using loss-free materials. We subsequently showed that the field is localized into a subwavelength $(\lambda / 8)$ region at the cavity resonance frequency. The proposed cavity structures can be extended to optical frequencies and can be used in several applications such as nonlinear optics [19] and high-density data storage.

This work is supported by the European Union (EU) under the projects EU-METAMORPHOSE, EU-PHOREMOST, EU-PHOME, EU-ECONAM, and TUBITAK under projects 105E066, 105A005, 106E198, 106A017. E. Ozbay also acknowledges partial support from the Turkish Academy of Sciences.

\section{References}

1. V. G. Veselago, Sov. Phys. Usp. 10, 504 (1968).

2. J. B. Pendry, A. J. Holden, D. J. Robbins, and W. J. Stewart, J. Phys. Condens. Matter 10, 4785 (1998).

3. J. B. Pendry, A. J. Holden, D. J. Robbins, and W. J. Stewart, IEEE Trans. Microwave Theory Tech. 47, 2075 (1999).
4. D. R. Smith, W. J. Padilla, D. C. Vier, S. C. NematNasser, and S. Schultz, Phys. Rev. Lett. 84, 4184 (2000).

5. E. Ozbay, I. Bulu, and H. Caglayan, Phys. Status Solidi B 244, 1202 (2007).

6. I. Bulu, H. Caglayan, and E. Ozbay, Opt. Lett. 31, 814 (2006).

7. D. Schurig, J. J. Mock, B. J. Justice, S. A. Cummer, J. B. Pendry, A. F. Starr, and D. R. Smith, Science 314, 977 (2006)

8. A. M. Belyantsev and A. B. Kozyrev, Tech. Phys. 47, 1477 (2002).

9. J. D. Joannopoulos, R. D. Meade, and J. N. Winn, Photonic Crystal: Molding the Flow of Light (Princeton U. Press, 1995).

10. M. Bayindir, B. Temelkuran, and E. Ozbay, Phys. Rev. Lett. 84, 2140 (2000).

11. P. A. Postigo, A. R. Alija, L. J. Martínez, M. L. Dotor, D. Golmayo, J. Sánchez-Dehesa, C. Seassal, P. Viktorovitch, M. Galli, A. Politi, M. Patrini, and L. C. Andreani, Photonics Nanostruct. Fundam. Appl. 5, 79 (2007).

12. P. R. Villenevue, S. Fan, and J. D. Joannopoulos, K.-Y. Lim, G. S. Petrich, L. A. Kolodziejski, and R. Reif, Appl. Phys. Lett. 67, 167 (1995).

13. P. L. Gourley, J. R. Wendt, G. A. Vawter, T. M. Brennan, and B. E. Hammons, Appl. Phys. Lett. 64, 687 (1994).

14. Y. H. Chen, J. W. Dong, and H. Z. Wang, Appl. Phys. Lett. 89, 141101 (2006).

15. K. Guven, K. Aydin, and E. Ozbay, Photonics Nanostruct. Fundam. Appl. 3, 75 (2005).

16. K. Aydin and E. Ozbay, IEE Proc. Microwaves, Antennas Propag. 1, 89 (2007).

17. E. Ozbay, K. Aydin, E. Cubukcu, and M. Bayindir, IEEE Trans. Antennas Propag. 51, 2592 (2003).

18. H. Caglayan, I. Bulu, M. Loncar, and E. Ozbay, Opt. Express 16, 11132 (2008).

19. A. Yariv and P. Yeh, Photonics: Optical Electronics in Modern Communications (Oxford U. Press, 2007). 